\title{
Using stellar population studies to determine the progenitors of GRBs and SNe
}

\author{
Christina C. Thöne ${ }^{1}$, Lise Christensen ${ }^{2}$ and Johan P. U. Fynbo ${ }^{3}$ \\ ${ }^{1}$ Istituto Nazionale di Astrofisica, Osservatorio Astronomico di Brera, Via E. Bianchi 46, \\ 23807 Merate, Italy \\ email: christina.thoene@brera.inaf.it \\ ${ }^{2}$ European Southern Observatory, K. Schwarzschild Str. 1, 85748 Garching, Germany \\ ${ }^{3}$ Dark Cosmology Centre, Niels Bohr Institut, University of Copenhagen, Juliane Maries Vej \\ 30, 2100 Copenhagen, Denmark
}

\begin{abstract}
We present spatially resolved emission line studies of three nearby GRB and SN hosts with longslit and/or IFU observations. We compare the environment of the GRBs/SNe with those of other star-forming regions in the host galaxy and try to get informations on the progenitor from stellar population models and metallicities.
\end{abstract}

Keywords. galaxies:abundances, gamma-rays: bursts, stars: supernovae: general

\section{Introduction}

Supernovae (SNe) and (long) Gamma-Ray Bursts (GRBs) are both end stages of a very massive star with GRBs likely requiring special conditions to occur. Long GRBs have also shown to produce supernovae, all of Type Ic. Whereas the progenitors of Type II SNe have been observed, we might never be able to see the progenitor of a GRB due to their larger distances. At most we can determine the properties of the stellar population in the environment of the GRB to get some hints on the progenitor. Theoretical studies of stellar populations in the local universe can help us in connecting our observations of GRB and SN sites to the properties of their stellar populations and their progenitors.

\section{What can we observe in GRB/SN hosts?}

The connection of GRBs and SNe with massive star formation places them preferentially in the brighter star-forming (SF) regions of their hosts. Studying the properties of the interstellar matter through emission lines is a well-known method and we can expect the composition of the stars inside those star-forming regions to be similar. One important ingredient in stellar evolution models is the metallicity which we can determine by emission line ratios such as the O3N2 and N2 parameters and GRBs have been found in lower metallicity regions than normal Type Ic SNe (Modjaz et al. 2008). The age can be determined by the EW of the Balmer lines or stellar population synthesis models and the Balmer line flux is connected to the star-formation rate. From the Balmer line decrement, we can furthermore determine the extinction in the line-of-sight.

\section{Results}

We present three nearby galaxies that hosted GRBs and/or SNe where we could study their resolved stellar populations. All three GRBs/SNe were rather special events from their high-energy perspective. GRB 980425 was a subluminous GRB with no optical 
afterglow. Some days later, a SN was observed coincident with the GRB position (Galama et al. (1998) providing the first evidence that GRBs are connected to Type Ic SNe. If present, a SN should have also been observed for GRB 060505 at $\mathrm{z}=0.008$, but no SN was detected to deep limits (e.g. Fynbo et al. 2006). Its relatively short duration suggested that it might belong to the short-GRB class supposed to originate from a compact object merger. SN 2008D finally was discovered first by a flash in X-rays attributed to the shock break-out of the SN (Soderberg et al. 2008) and later identified to be a SN Type Ib.
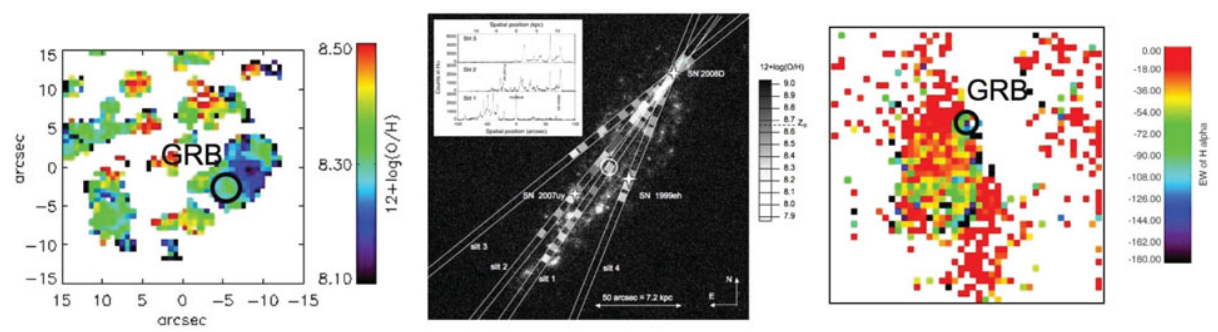

Figure 1. Resolved metallicities for the host of GRB 980425 and SN 2008D and H $\alpha$ EW for the host of GRB 060505.

The host of GRB 980425. This spiral dwarf galaxy at $\mathrm{z}=0.008$ studied with the VIMOS Integral-Field-Unit (IFU) had one very bright region with exceptional properties, also showing WR features, close to the GRB/SN site. The GRB site itself, another starforming region, does not show very different properties compared to the rest of the galaxy in terms of metallicity, age, star-formation rate and extinction. However, if this host had been at higher redshifts, the GRB and the WR region could not have been distinguished and lead to wrong conclusions about the properties of the GRB (Christensen et al. 2008). The host of GRB 060505. Triggered by the debate about the issue of the GRB progenitor, our first rough spatially resolved study with FORS longslit observations revealed that the region around the GRB site was rather different from the rest of the galaxy with higher SF rate and a rather low metallicity. This suggested that the GRB was despite its duration likely to the collapse of a massive star (Thöne et al. 2007). A refined study with VIMOS/IFU data confirms these results and the SF region around the GRB together with another nearby large SF region are the most exeptional places in the host galaxy.

NGC2770 - the host of 3 Type Ib SNe. The host of SN 2008D hosted in total 3 Type Ib SNe in the last 10 years. This lead us to investigate the properties of the 3 SN sites and compare them with the rest of the galaxy. All three SN sites show subsolar metallicity but a much lower SFR and higher age than found for GRBs connected to SN Type Ic, suggesting a different progenitor (Thöne et al. 2009). A refined study with VIMOS/IFU is currently on the way also for this galaxy.

Spatially resolved studies of GRB and SN hosts might be a promising tool to reveal some properties of unobserved and unobservable progenitor stars in particular for the more distant GRBs. However, we should be careful connecting global properties of galaxies with the properties of the immediate environment and hence the progenitor.

\section{References}

Galama, T. et al. 1998, Nature, 395, 670

Fynbo, J. et al. 2006, Nature, 444, 1047

Soderberg, A. et al. 2008, Nature, 454, 246

Modjaz, M. et al. 2008, AJ, 135, 1136
Christensen, L. et al. 2008, A\&A, 490, 45

Thöne et al. 2007, ApJ, 676, 1151

Thöne et al. 2009, ApJ, 698, 1207 